Ann. Biol. anim. Bioch. Biophys., 1977, 17 (2), 187-191.

\title{
Corrélation entre le poids de la glande pinéale, le poids soma- tique et le poids de l'encéphale chez les Artiodactyles
}

\author{
par Hermance LEGAIT, E. LEGAIT et Henriette OBOUSSIER \\ Laboratoire d'Histologie de la Faculté de Médecine A de Nancy \\ Route de Moron, B. P. 1080, 54019 Noncy Cedex, France \\ Zoologisches Institut und Zoologisches Museum Universität Hamburg \\ Martin-Luther-King Platz 3, 2000 Hamburg 13, Allemagne Fédérale
}

Summary. Correlation between pineal, somatic and brain weight in Artiodactyles.

The pineal gland of 30 species of Artiodactyles is weighed and compared to body weight and brain weight.

The correlation coefficients are good $(0.8592$ for the relation pineal/body weight and 0.8427 for the relation pineal/brain weight) and statistically significant $(\alpha=0.01)$. The epiphyseal indices vary from 17 to 250 because of the important interspecific variations observed in pineal weight.

The allometry coefficient is close to isometry for the relation pineal/somatic weight (0.8427) confirming previous results on the volume of the epiphysis in Insectivora, Prosimians and Simians. The allometry coefficient (1.3955) for the relation pineal/brain weight is higher, a fact that is to be considered consistant with the value of the relation brain weight/somatic weight $(0.607)$ with a correlation coefficient of 0.9831 .

\section{Introduction.}

Le poids de la glande pinéale n'a fait l'objet d'aucune étude statistique et mathématique chez les Artiodactyles. Madame le Professeur Oboussier possédant une importante collection de cerveaux d'Artiodactyles, il nous a été possible de poursuivre notre étude morphométrique consacrée à la glande pinéale des Mammifères et commencée chez les Rongeurs, les Chiroptères, les Insectivores, les Lémuriens, les Simiens et l'Homme $(1,2,3,4)$. Ces premières recherches nous avaient permis de montrer que le volume de la glande pinéale présente de grandes variations individuelles à l'intérieur d'une même espèce à poids somatique constant ainsi que de grandes variations interspécifiques à l'intérieur de chaque Ordre. Le volume de l'hypophyse et des lobes hypophysaires $(1,3,4)$ présente des variations beaucoup plus faibles. Nous en avions conclu que la glande pinéale n'esł pas liée à une fonction physiologique essentielle (comme c'est le cas pour l'hypophyse). Il est bien connu par exemple que le Rat peut survivre longtemps à une épiphysectomie mais survit plus difficilement après une hypophysectomie. 


\section{Matériel et méthodes.}

L'étude porte sur 30 espèces d'Artiodactyles provenant de différents pays, chaque espèce étant représentée par 1 à 9 animaux, soit 79 animaux.

Le poids somatique a pu être établi le plus souvent par pesée. Cependant cette mesure n'a pu être effectuée dans un certain nombre de cas ef notamment chez les animaux de poids très élevé. Dans ces cas, un poids approximatif (ca) a été utilisé.

Le poids de l'encéphale, extrait du cerveau après section des nerfs crâniens ef du tronc cérébral entre le myélencéphale et la moelle épinière, a pu être établi dans tous les cas aussitôt après le sacrifice de l'animal. Les encéphales ont ensuite été conservés dans le formol à 10 p. 100.

Les glandes pinéales prélevées, par section de la commissure habénulaire et de la commissure blanche postérieure sur les cerveaux fixés, ont été pesées individuellement.

TABLEAU 1

\begin{tabular}{|c|c|c|c|c|c|}
\hline Espèce & Nombre & $\begin{array}{c}\text { Poids } \\
\text { somatique } \\
\text { en kg }\end{array}$ & $\begin{array}{l}\text { Poids } \\
\text { encéphalique } \\
\text { en } g\end{array}$ & $\begin{array}{l}\text { Epiphyse } \\
\text { en } g\end{array}$ & $\begin{array}{c}\text { Indice } \\
\text { épiphysaire }\end{array}$ \\
\hline 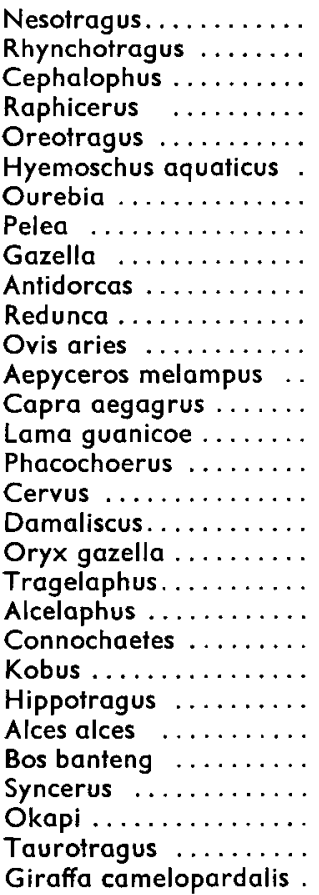 & $\begin{array}{l}1 \\
2 \\
5 \\
2 \\
1 \\
1 \\
1 \\
2 \\
2 \\
2 \\
7 \\
1 \\
1 \\
1 \\
1 \\
2 \\
1 \\
7 \\
2 \\
9 \\
7 \\
3 \\
8 \\
2 \\
2 \\
1 \\
1 \\
1 \\
2 \\
1\end{array}$ & $\begin{array}{c}3,300 \\
4,815 \\
8,600 \\
10,700 \\
11 \\
12 \\
12,5 \\
22,75 \\
29,9 \\
30 \\
42 \\
\text { Ca } 46 \\
\text { Ca } 50 \\
\text { Ca } 50 \\
73 \\
76 \\
105 \\
115 \\
127,5 \\
138 \\
148,5 \\
157 \\
167 \\
195 \\
205 \\
\text { Ca } 260 \\
\text { Ca } 300 \\
\text { Ca } 320 \\
\mathrm{Ca} 390 \\
\mathrm{Ca} 470\end{array}$ & $\begin{array}{c}30 \\
30,5 \\
74,4 \\
53 \\
59,5 \\
\mathrm{Ca} \quad 69 \\
55 \\
115 \\
91,5 \\
130 \\
123 \\
147 \\
185 \\
160 \\
235 \\
146 \\
238,5 \\
285 \\
255,5 \\
268 \\
287 \\
334 \\
257 \\
340 \\
359 \\
417 \\
378 \\
475 \\
537 \\
595\end{array}$ & $\begin{array}{l}0,0089 \\
0,0076 \\
0,0148 \\
0,0194 \\
0,0263 \\
0,0099 \\
0,0137 \\
0,0325 \\
0,0368 \\
0,0294 \\
0,0389 \\
0,0477 \\
0,0097 \\
0,0267 \\
0,0874 \\
0,0465 \\
0,2445 \\
0,0928 \\
0,1350 \\
0,0893 \\
0,1323 \\
0,4254 \\
0,0488 \\
0,0813 \\
0,1484 \\
0,1578 \\
0,3345 \\
0,0599 \\
0,1550 \\
0,3609\end{array}$ & $\begin{array}{r}251 \\
146 \\
159 \\
167 \\
220 \\
76 \\
101 \\
131 \\
112 \\
89 \\
84 \\
94 \\
18 \\
49 \\
107 \\
55 \\
218 \\
73 \\
95 \\
58 \\
80 \\
244 \\
28 \\
37 \\
65 \\
54 \\
100 \\
17 \\
35 \\
68\end{array}$ \\
\hline
\end{tabular}


L'étude statistique de toutes nos données chiffrées a été effectuée à l'aide de la Programma 101 du laboratoire d'informatique de la Faculté de Médecine de Nancy (Prof. Martin).

Nous avons calculé : 1) le coefficient de corrélation liant le poids de l'épiphyse au poids somatique et au poids encéphalique $(r), 2$ ) le pourcentage de variance de la variable dépendante, attribuable aux variations de la variable indépendante ( $\left.r_{2} p .100\right)$, 3) un coefficient d'allométrie, l'axe majeur réduit (AMR), 4) les indices épiphysaires permettant la comparaison de poids épiphysaires d'espèces distinctes, indépendamment de leur poids somatique (tabl. 1).

\section{Observations.}

La position de la glande pinéale des Artiodactyles ne présente aucune particularité aigne d'être signalée. Signalons cependant que chez Giraffa camelopardalis (comme chez un Périssodactyle que nous avons pu étudier, Equus hemionus onager), la glande pinéale se trouve située au fond d'un entonnoir ; les deux hémisphères ne sont pas accolés sur toute leur longueur comme c'est le cas chez la plupart des Mammifères, mais s'écartent légèrement l'un de l'autre au-dessus de la glande pinéale qui se trouve ainsi située au fond d'un puits. Les hémisphères cérébraux sont accolés en avant ef en arrière de ce puits. Nous avons pu étudier trois cerveaux de Girafe.

La forme de l'épiphyse est variable : parfois arrondie (chez le Lama, elle ressemble à un pois chiche) mais le plus souvent un peu allongée. Chez Cervus, cette glande est pigmentée mais ce n'est pas le cas pour les autres espèces étudiées.

\section{Résultats.}

1) Relation poids de l'épiphyse/poids somatique Ep/Ps (fig. 1).

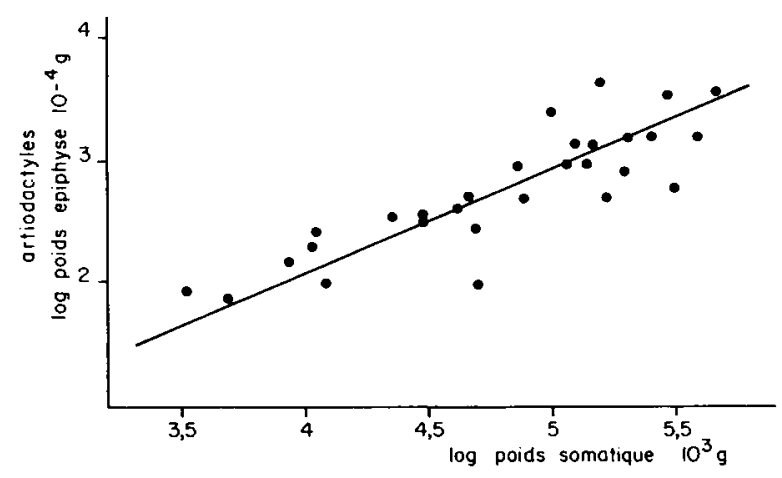

FIG. 1.

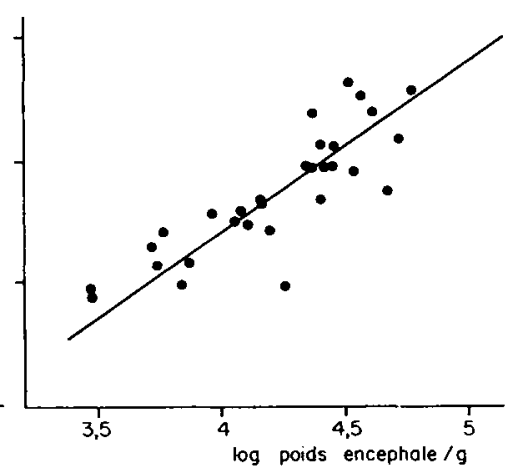

FIG. 2.

FIG. 1. - Relation poids de l'épiphyse/poids somatique en doubles coordonnées logorithmiques chez les Artiodactyles.

FIG. 2. - Relation poids de l'épiphyse/poids encéphalique en doubles coordonnées logarithmiques chez les Artiodactyles. 
Les valeurs obtenues sont les suivantes:

$\begin{array}{cccc}r & r^{2} \text { p. } 100 & \text { AMR } & n \\ 0,8592 & 74 & 0,8427 & 30\end{array}$

$\mathrm{n}=$ nombre d'espèces étudiées.

Le coefficient de corrélation est élevé (différent de $0: p<1$ p. 100). Le coefficient d'allométrie se rapproche de l'isométrie. Ces résultats sont comparables à ceux que nous avons obtenus précédemment dans les autres Ordres de Mammifères étudiés et notamment dans le groupe comprenant les Insectivores, les Lémuriens et les Simiens (le plus homogène) dont le coefficient d'allométrie volume épiphysaire/poids somatique était de 1,009.

2) Relation poids de l'épiphyse/poids encéphalique Ep/Pe (fig. 2).

Les valeurs obtenues sont les suivantes:

$\begin{array}{cccc}r & r^{2} \text { P. } 100 & \text { AMR } & n \\ 0,8496 & 72 & 1,3955 & 30\end{array}$

Le coefficient de corrélation de la relation $E p / P s$ est légèrement plus élevé que celui de la relation $\mathrm{E}_{\mathrm{p}} /$ Penc, ce que nous avons également observé dans les autres ordres de Mammifères étudiés.

Le poids de l'épiphyse est plus intimement corrélé au poids somatique qu'au poids encéphalique.

Le coefficient d'allométrie est plus élevé ce qui est fréquemment le cas puisque les allométries $\mathrm{Pe} / \mathrm{Ps}$ sont souvent voisines de $2 / 3$ ( 0,607 pour nos Artiodactyles avec un coefficient de corrélation de 0,9831 ).

3) Indices épiphysaires.

Le tableau 1 contient également pour chaque espèce, les indices épiphysaires qui sont les valeurs $k$ de la formule :

$$
\text { Poids épiphysaire }=k \cdot P^{1,009}
$$

(le coefficient 1,009 correspondant au coefficient d'allométrie de la relation Ep/Ps chez les Insectivores, les Lémuriens ef les Simiens).

De plus, les valeurs $k$ ainsi calculées ont été rapportées en pourcentage de la valeur $k_{0}$ qui définit l'axe majeur réduit de ces Mammifères. L'intérêt de tels indices (dont la valeur est relative étant donné le petit nombre d'animaux de chaque espèce) est de permettre la comparaison instantanée des poids épiphysaires d'espèces distinctes, indépendamment de leur poids somatique. Ainsi il nous est possible de montrer que, parmi les Artiodactyles, c'est l'Okapi qui possède la plus petite épiphyse et Nesotragus ainsi que Connochaetes la plus grosse. La valeur moyenne de ces indices est 100 et l'écart-type est 65 . La forte valeur de cet écart-type traduit la grande variabilité des poids épiphysaires.

Notons que l'étude des volumes épiphysaires chez les Insectivores, les Lémuriens, les Simiens, les Rongeurs et les Chiroptères nous avait également fourni des résultats semblables. 
Les différences intraspécifiques de poids épiphysaires sont aussi grandes que les différences interspécifiques. Citons par exemple le cas de 2 Redunca mâles de $45 \mathrm{~kg}$ dont l'encéphale pèse $117 \mathrm{~g}$ dans les 2 cas et qui possèdent des épiphyses dont le poids va du simple au double : $0,0142 \mathrm{~g}$ et $0,0353 \mathrm{~g}$.

Accepté en octobre 1976.

\section{Références}

1. LEGAIT H. et BAUCHOT R. Etude des corrélations liant les volumes de la glande pinéale, de l'OSF ef des lobes hypophysaires au poids somatique et au volume de l'hypothalamus chez les Rongeurs. C. R. Soc. Biol. (à paraître).

2. LEGAIT H., BAUCHOT R. et STEPHAN H. Etude statistique des corrélations liant le volume de l'épiphyse au poids somatique et au poids de l'encéphale chez les Rongeurs, les Insectivores, les Chiroptères, les Lémuriens et les Simiens. Mammalia (à paraître).

3. LEGAIT H., MUR J. M., BAUCHOT R. 1976. Corrélations liant les volumes hypophysaires et épiphysaires au poids somatique ef au poids encéphalique chez les Simiens ef l'Homme. C. R. Soc. Biol., 170, 870-879.

4. LEGAIT H., MUR J. M., BAUCHOT R. ef CONTET-AUDONNEAU J. L. Etude des corrélations liant les volumes des lobes hypophysaires et de l'épiphyse au poids somatique et au poids de l'encéphale chez les Chiroptères. Bull. Ass. Anat. (à paraître). 